\title{
SVW Vascular \\ Prestroke selective serotonin reuptake inhibitor use and functional outcomes after ischaemic stroke
}

\author{
Mark R Etherton, ${ }^{1,2}$ Khawja A Siddiqui, ${ }^{2}$ Lee H Schwamm ${ }^{1,2}$
}

To cite: Etherton MR, Siddiqui KA, Schwamm LH. Prestroke selective serotonin reuptake inhibitor use and functional outcomes after ischaemic stroke. Stroke and Vascular Neurology 2018;3: e000119. doi:10.1136/svn2017-000119

- Additional material is published online only. To view please visit the journal online (http://dx.doi.org/10.1136/svn2017-000119).

Received 20 0ctober 2017 Revised 1 December 2017 Accepted 24 December 2017 Published Online First 13 January 2018
Check for updates

'Department of Neurology, Harvard Medical School, Boston, Massachusetts, USA

${ }^{2}$ Department of Neurology, Massachusetts General Hospital, Boston, Massachusetts, USA

Correspondence to

Dr Mark R Etherton;

metherton@partners.org

\section{ABSTRACT}

Background Selective serotonin reuptake inhibitors (SSRIs) have been implicated in contributing to recovery after acute ischaemic stroke. In particular, poststroke initiation of an SSRI has been demonstrated to improve motor recovery. The role of prestroke SSRI use on functional outcomes and stroke recovery is less clear. We aimed to examine the effect of prestroke SSRI use on metrics of hospitalisation and functional recovery. Methods We included 4968 consecutive patients from January 2006 to June 2015 in our local Get With The Guidelines-Stroke registry in whom a preadmission drug list could be extracted from an administrative research data registry. Univariate and multivariate analyses were performed to identify predictors of functional outcomes. Results On univariate analysis, among 4698 ischaemic strokes (740 SSRI users and 3948 non-users), SSRI use before acute ischaemic stroke did not impact the National Institutes of Health Stroke Scale (NIHSS) admission score, length of stay or rate of symptomatic haemorrhage. Patients using SSRIs prior to their stroke were more likely to present with weakness $(57 \%$ vs $47.3 \% ; \mathrm{P}<0.001)$ and have hospitalisations complicated by pneumonia $(7.6 \%$ vs $5.7 \% ; P<0.001)$. Moreover, prestroke SSRI use was associated with a negative impact on ambulatory status at discharge and discharge to home. On multivariate regression analysis, SSRI use was associated with lower likelihood of discharge to home (adjusted OR $0.79,95 \% \mathrm{Cl}$ 0.62 to $0.997, P<0.05$ )

Conclusions SSRI use preceding an acute ischaemic stroke is associated with lower rates of discharge to home despite no significant increase in length of stay or NIHSS score.

\section{INTRODUCTION}

The management of acute ischaemic stroke (AIS) has been revolutionised by the availability of intravenous thrombolysis and endovascular approaches. ${ }^{1-4}$ Despite these advances in effective reperfusion strategies, AIS remains a significant cause of disability. Of stroke survivors, two out of three will have residual neurological impairment, and more than $25 \%$ are dependent on activities of daily living. ${ }^{56}$ The economic repercussions of poststroke disability are enormous; in the USA alone, the total annual cost exceeds $\$ 36$ billion. ${ }^{7}$ This burden of disease and healthcare expenditures has been the impetus for investigational research targeting strategies for supplementing poststroke recovery.

One such area has been the administration of selective serotonin reuptake inhibitors (SSRIs) following AIS, which have been shown to positively modulate motor recovery and decrease disability even after adjustment for comorbid mood disorders. ${ }^{8-12}$ In animal studies, several beneficial effects have been attributed to SSRIs, including reducing infarct volume, stimulating neurogenesis, upregulating anti-inflammatory effects, enhancing cerebral autoregulation and increasing neuroplasticity. ${ }^{10-17}$ At the molecular level, the effects on neuronal plasticity in part appear to be due to SSRI-mediated modulation of brain-derived neurotrophic factor (BDNF) expression. ${ }^{18}$ Furthermore peristroke administration of BDNF in rat models of middle cerebral artery occlusion decreased infarct volume and improved functional recovery. ${ }^{19}$

In the prestroke and peristroke periods in humans, however, the implications of SSRI treatment on functional recovery remain less clear. A recent observational study of prestroke SSRI (preSSRI) use showed no effect on either stroke severity or mortality in AIS. ${ }^{20}$ In contrast, a small, retrospective comparison of prestroke versus poststroke SSRI treatment suggested pretreatment was associated with favourable functional outcomes and change in score on the National Institutes of Health Stroke Scale (NIHSS) at discharge. ${ }^{9}$ These findings highlight the need for additional clinical studies that assess the influence of preSSRI use on functional outcomes. In this study, we evaluated preSSRI use and the relationship with several markers of functional status at discharge in patients admitted with AIS. We also repeated these analyses for patients taking any type of antidepressant medication prior to admission to assess for the presence of any drug class effects. 


\section{METHODS}

\section{Study population}

The details of case ascertainment and data collection of the Get With The Guidelines-Stroke (GWTG-Stroke) registry at Massachusetts General Hospital (MGH) are described elsewhere. ${ }^{21} 22$ Briefly, we included data from 4698 patients admitted to MGH between 1 January 2006 and 30 April 2014 with a diagnosis of AIS. Trained hospital personnel were instructed in using the GWTGStroke registry to collect data on consecutive patients admitted for AIS. Patients were included by a combination of prospective clinical identification or retrospectively via the International Classification of Diseases-Ninth Revision discharge codes.

In this study, patients were included if they (1) were discharged from MGH between 1 January 2006 and 30 April 2014 with AIS as their primary hospitalisation reason; (2) were entered into our GWTG-Stroke registry; and (3) had medication reconciliation documented on admission. The preSSRI group was composed of patients taking various SSRI preparations, including citalopram, escitalopram, fluoxetine, paroxetine and sertraline. We then conducted a sensitivity analysis to evaluate the association between the outcomes of interest and any antidepressant use (preAD), and identified patients taking bupropion, duloxetine, venlafaxine, amitriptyline and nortriptyline. Patients were excluded if there was no 'prior to admission' medication list documented in their chart.

\section{Variables of interest}

In this study, we performed regression analysis to evaluate the association of preSSRI or any antidepressant use (preAD) with surrogates of stroke severity and functional status at discharge. Preadmission medications were extracted for all patients using hospital records. A list was then generated that included all medications being used by the patients in the cohort. A trained physician then categorised the drugs into SSRIs, other antidepressants or not antidepressant groups. Patients were assigned to the SSRI or antidepressant group if they were actively taking one of the specified agents, whether it was prescribed for reasons other than mood disorder (eg, peripheral neuropathy, migraine).

Patients' demographic and socioeconomic measures included age, sex, race (Asian, African-American/black, white or other), Hispanic ethnicity, marital status and insurance status (private/other, Medicare, Medicaid, uninsured/self pay). Medical history was obtained from self-reports or electronic records, and included the presence of depression as well as risk factors for stroke: atrial fibrillation, coronary artery disease or prior myocardial infarction, carotid stenosis, diabetes mellitus, dyslipidaemia, heart failure, hypertension, peripheral vascular disease, previous stroke/transient ischaemic attack (TIA), renal insufficiency, or smoking. Medical history of depression was abstracted from the medical record physician notes, whereas antidepressant class medication use was taken from medication reconciliation data without requiring linkage to a diagnosis of depression.

The characteristics of the symptoms on initial presentation, hospital course and status at discharge were also analysed. On presentation, the initial NIHSS score and the presenting exam findings (weakness, aphasia, altered mental status or other) were recorded. Variables pertaining to the hospital course included length of stay, hospital-acquired pneumonia, urinary tract infection, use of intravenous tissue plasminogen activator (tPA) or intra-arterial thrombolysis or thrombectomy, and symptomatic haemorrhage. Unless specified as occurring at an outside referring hospital, the laboratory values measured and any therapies delivered were at our hospital. The results of laboratory studies evaluating the serum lipid panel, haemoglobin A1c, fasting blood glucose level and international normalised ratio were compared between groups. At discharge, the variables of interest included a three-level assessment of ambulatory status at discharge (able to ambulate independently with or without a device, ambulate with assistance or unable to ambulate) and discharge disposition (home, rehabilitation facility, skilled nursing facility, expired/hospice or other).

\section{Statistical analysis}

Descriptive statistics were performed to compare differences in demographic, clinical characteristics, AIS care and functional status at discharge between patients with preSSRI use and those on no antidepressants. This was then repeated for patients on any type of antidepressant versus no antidepressants. Means, SD and percentages, or median and IQRs were generated for each variable. Two-way t-tests (for normally distributed variables), Wilcoxon rank-sum (for non-normally distributed variables) and $\chi^{2}$ tests (for categorical variables) were performed to determine associations. Statistical significance was set at the $\mathrm{P}=0.05$ level.

We then performed stepwise logistic regression models to examine the association between the outcome of interest (ie, discharge to home) and preSSRI, adjusting for covariates known to predict stroke care and outcomes. This was then repeated for patients on any type of antidepressant versus no antidepressants. All variables with $\mathrm{P}<0.1$ on univariate testing were added to the model, along with key items of interest (eg, depression history) and those previously shown to predict stroke outcome (eg, ambulatory status). The multivariable analyses were done with two adjustments: first, for variables available on admission (partially adjusted model); and second, for all admission variables plus treatments/complications (final adjusted model). ORs and 95\% CIs were calculated for each covariate. The univariate comparisons for SSRI and antidepressant use are shown as percentages or means/ medians in table 1 and online supplementary table 1 , respectively. The analyses of discharge status are reported in table 2 and online supplementary table 2 as unadjusted univariate ORs and multivariable adjusted ORs (aOR). 
Table 1 Univariate analysis of baseline characteristics, inhospital treatments, medical complications and discharge outcomes among patients with prestroke SSRI use

\begin{tabular}{|c|c|c|c|}
\hline & SSRI use $n=740$ & No SSRI use $n=3741$ & $P$ value \\
\hline \multicolumn{4}{|l|}{ Demographics } \\
\hline Age, years, mean (SD) & $68.24(14.52)$ & $68.58(15.45)$ & 0.58 \\
\hline Female gender (\%) & 55.7 & 42.5 & $<0.001$ \\
\hline Length of stay, days, mean (SD) & $6.34(5.59)$ & $6.10(6.74)$ & 0.37 \\
\hline Location at stroke onset (\%) & & & 0.01 \\
\hline Not in a healthcare setting & 86.5 & 85.5 & \\
\hline Acute care facility & 5.5 & 8.6 & \\
\hline Chronic healthcare facility & 3.0 & 1.9 & \\
\hline Outpatient setting & 0.4 & 0.6 & \\
\hline After hospital arrival & 2.4 & 1.8 & \\
\hline ND & 2.2 & 1.4 & \\
\hline Race (\%) & & & 0.002 \\
\hline White & 87.4 & 83.7 & \\
\hline African-American & 4.2 & 6.1 & \\
\hline Asian & 1.8 & 4.1 & \\
\hline Other & 6.6 & 6.0 & \\
\hline Hispanic ethnicity (\%) & 5.5 & 5.4 & 0.74 \\
\hline Insurance (\%) & & & 0.29 \\
\hline Medicare & 55.7 & 54.5 & \\
\hline Medicaid & 2.0 & 1.3 & \\
\hline Private & 40.3 & 41.0 & \\
\hline Self-pay & 1.9 & 2.9 & \\
\hline \multicolumn{4}{|l|}{ Medical history (\%) } \\
\hline No medical history & 8.2 & 11.1 & 0.02 \\
\hline Atrial fibrillation & 19.5 & 20.7 & 0.46 \\
\hline Coronary artery disease & 21.6 & 20.9 & 0.67 \\
\hline Carotid stenosis & 3.1 & 3.7 & 0.44 \\
\hline Depression & 4.7 & 0.5 & $<0.001$ \\
\hline Diabetes mellitus & 28.8 & 23.2 & 0.001 \\
\hline Drugs/Alcohol & 2.3 & 1.3 & 0.05 \\
\hline Dyslipidaemia & 46.9 & 42.9 & 0.05 \\
\hline Family history of stroke & 2.6 & 1.4 & 0.02 \\
\hline Heart failure & 6.4 & 6.3 & 0.96 \\
\hline Hypertension & 73.2 & 69.0 & 0.02 \\
\hline Previous stroke & 21.5 & 12.6 & $<0.001$ \\
\hline Previous transient ischaemic attack & 6.9 & 4.1 & 0.001 \\
\hline Renal insufficiency & 3.8 & 3.4 & 0.56 \\
\hline Smoker & 13.4 & 12.9 & 0.70 \\
\hline \multicolumn{4}{|l|}{ Clinical characteristics } \\
\hline Ambulate at admission (\%) & 84.3 & 86.8 & 0.08 \\
\hline Heart rate, bpm, mean (SD) & $79.02(17.47)$ & $79.23(17.57)$ & 0.80 \\
\hline NIHSS, median (IQR) & $5(2-10)$ & $4(1-11)$ & 0.06 \\
\hline \multicolumn{4}{|l|}{ Initial exam finding (\%) } \\
\hline Weakness & 57.0 & 47.3 & $<0.001$ \\
\hline
\end{tabular}


Table 1 Continued

\begin{tabular}{|c|c|c|c|}
\hline & SSRI use $n=740$ & No SSRI use $n=3741$ & $P$ value \\
\hline Aphasia & 30.7 & 24.7 & 0.001 \\
\hline Altered mental status & 12.4 & 12.2 & 0.82 \\
\hline Other neurological symptoms & 15.1 & 14.1 & 0.48 \\
\hline \multicolumn{4}{|l|}{ Admission laboratory data, mean (SD) } \\
\hline Cholesterol, mg/dL & $166.57(47.73)$ & $165.33(48.25)$ & 0.53 \\
\hline Triglyceride, mg/dL & $127.94(74.54)$ & $123.75(77.44)$ & 0.19 \\
\hline High-density lipoprotein, mg/dL & $45.75(15.66)$ & $45.84(15.19)$ & 0.89 \\
\hline Low-density lipoprotein, mg/dL & $94.09(35.59)$ & $94.54(37.44)$ & 0.77 \\
\hline Haemoglobin A1c, \% & $6.21(1.24)$ & $6.13(1.23)$ & 0.15 \\
\hline Fasting blood glucose, mg/dL & $132.57(49.23)$ & $131.95(50.31)$ & 0.79 \\
\hline International normalised ratio & $1.20(0.59)$ & $1.18(0.64)$ & 0.65 \\
\hline \multicolumn{4}{|l|}{ Treatments and complications (\%) } \\
\hline Intravenous tPA at our hospital & 5.7 & 6.4 & 0.43 \\
\hline Intravenous tPA outside our hospital & 18.4 & 15.0 & 0.02 \\
\hline Intra-arterial therapy & 4.7 & 4.6 & 0.95 \\
\hline Symptomatic intracranial haemorrhage & 0.7 & 1.4 & 0.12 \\
\hline Pneumonia & 7.6 & 5.7 & $<0.001$ \\
\hline Urinary tract infection & 7.2 & 7.8 & $<0.001$ \\
\hline Ambulatory status at discharge & & & $<0.001$ \\
\hline Ambulate independently & 40.4 & 50.5 & \\
\hline Ambulate with assistance & 41.1 & 31.0 & \\
\hline Unable to ambulate & 12.2 & 11.5 & \\
\hline ND & 6.3 & 7.1 & \\
\hline Discharge destination & & & $<0.001$ \\
\hline Home & 37.7 & 48.4 & \\
\hline Rehabilitation facility & 46.1 & 36.1 & \\
\hline Skilled nursing facility & 6.6 & 4.1 & \\
\hline Expired/Hospice & 7.2 & 8.8 & \\
\hline Other & 2.4 & 2.6 & \\
\hline
\end{tabular}

bpm, beats per minute; ND, not determined; NIHSS, National Institutes of Health Stroke Scale; SSRI, selective serotonin reuptake inhibitors; tPA, tissue plasminogen activator.

\section{RESULTS}

A total of 4698 patients with AIS met the study inclusion/ exclusion criteria. Among these 4698 subjects, $19.5 \%$ $(n=920)$ were in the preAD group and $15.8 \%(n=740)$ were in the preSSRI group, with $80.4 \%$ of those subjects on antidepressants using an SSRI. Overall, our population comprised $45 \%$ women, predominately white $(85 \%)$ and a mean age of 68 years. In our population, $20.8 \%$ of the subjects had a prior stroke or TIA and $6.4 \%$ a diagnosis of depression. At the time of admission, $86.4 \%$ of our population was ambulatory.

\section{PreSSRI use and outcomes}

On univariate analysis, there were significantly higher rates in the preSSRI group of antecedent depression, diabetes, hypertension and prior stroke/TIA (all $\mathrm{P}<0.01$ ) (table 1), but no difference in rates of administration of inhospital intravenous tPA or intra-arterial thrombolysis, rates of symptomatic haemorrhage or length of stay. Women were also more likely to be on an SSRI prior to hospitalisation $(55.7 \%$ vs $42.5 \%$; $\mathrm{P}<0.001)$. There was a higher rate of intravenous tPA use at an outside referring hospital prior to transfer $(18.4 \%$ vs $15.0 \% ; \mathrm{P}=0.02)$ and a trend towards higher initial stroke severity (median NIHSS 5 vs 4; $\mathrm{P}=0.06$ ) (table 1 ). Patients in the preSSRI group were more likely to present with weakness (57\% vs $47.3 \% ; \mathrm{P}<0.001)$ and aphasia $(30.7 \%$ vs $24.7 \% ; \mathrm{P}=0.001)$ and have hospitalisations complicated by pneumonia (7.6\% vs $5.7 \%$; $\mathrm{P}<0.001$ ) (table 1$)$.

We then evaluated the factors pertaining to functional status at discharge. Univariate analysis of the preSSRI group showed a negative association with ambulatory status at discharge $(40.4 \%$ vs $50.5 \% ; \mathrm{P}<0.001)$ and 
Table 2 Multivariable analysis of clinical factors associated with home discharge after hospitalisation for acute ischaemic stroke

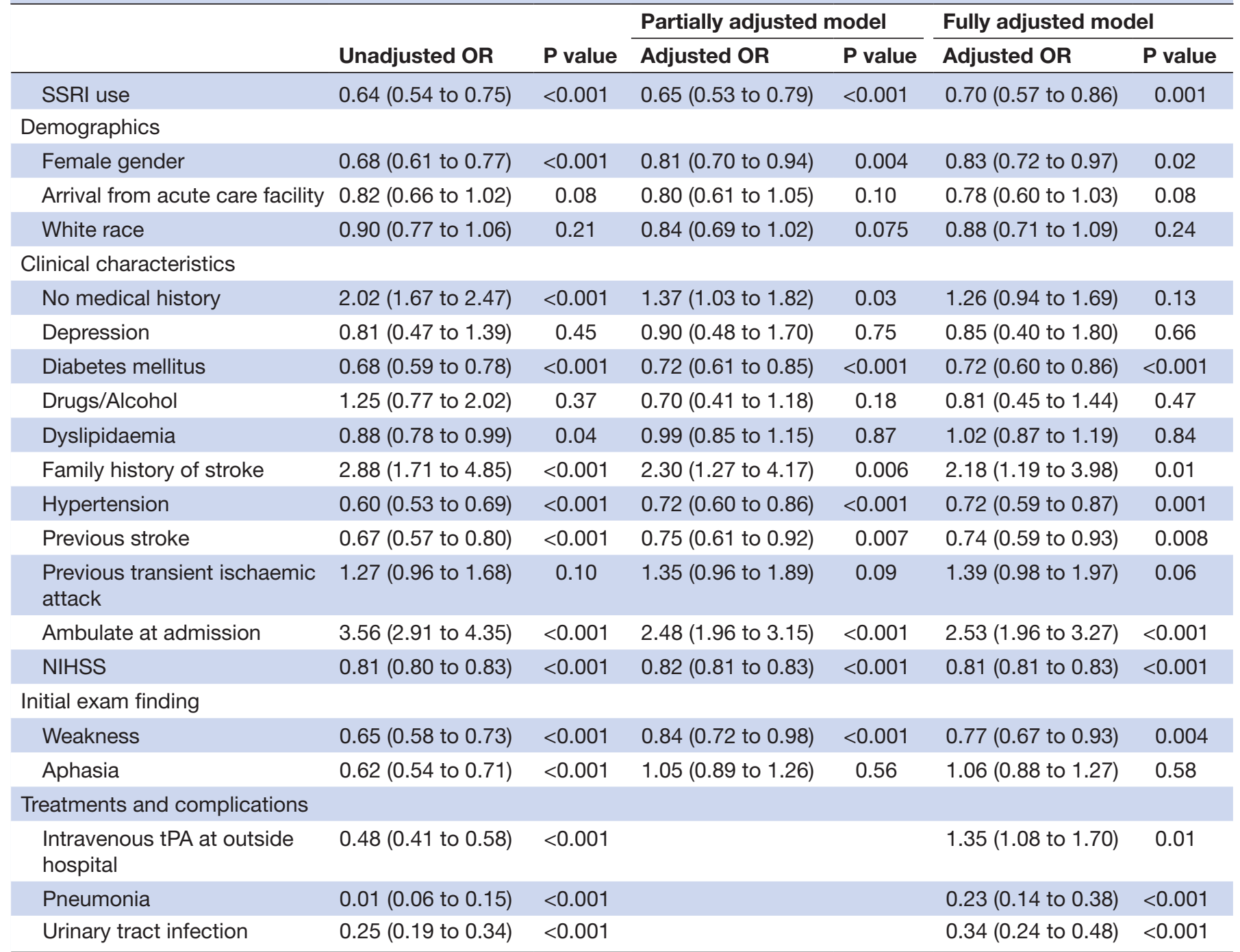

NIHSS, National Institutes of Health Stroke Scale; SSRI, selective serotonin reuptake inhibitors; tPA, tissue plasminogen activator.

discharge to home $(37.7 \%$ vs $48.4 \%$; $\mathrm{P}<0.001)$ (table 1$)$. The multivariable analysis was performed with two adjustments: first, in the partially adjusted model, all variables available on admission with $\mathrm{P}<0.1$ on univariate analysis plus factors known to impact stroke outcomes (eg, depression, ambulatory status) were included; and second, the final adjusted model, which included all variables from the partially adjusted model plus significant treatment/ outcome variables identified on univariate analysis (table 2). On multivariable regression analysis, SSRI use was independently associated with a lower likelihood of discharge to home in both the partially adjusted model (aOR $0.65,95 \%$ CI 0.53 to $0.79, \mathrm{P}<0.001$ ) and the fully adjusted model (aOR $0.70,95 \%$ CI 0.57 to $0.86, \mathrm{P}=0.001$ ) (table 2). In the fully adjusted model, female gender, arrival from an acute care facility, diabetes, hypertension, prior stroke, NIHSS score, weakness on initial exam, and hospitalisations complicated by pneumonia or urinary tract infection decreased the odds of discharge to home (table 2). The ability to ambulate on admission (aOR 2.53,
95\% CI 1.96 to $3.27, \mathrm{P}<0.001)$ and administration of intravenous tPA at a hospital prior to transfer to our facility (aOR $1.35,95 \%$ CI 1.08 to $1.70, \mathrm{P}=0.01$ ) were associated with increased odds of discharge to home (table 2).

\section{Prestroke antidepressant use of all types and outcomes}

To determine if these associations observed with preSSRI use and functional status at discharge were a class effect or persisted in patients using other forms of antidepressants (preAD), we pursued a sensitivity analysis including all patients regardless of type of antidepressant.

Similar to the preSSRI group, univariate analysis of the preAD group showed a negative association with ambulatory status at discharge $(43.8 \%$ vs $50.3 \% ; \mathrm{P}=0.01)$ and discharge to home $(39.7 \%$ vs $48.5 \%$; $\mathrm{P}<0.001)$ (online supplementary table 1 ). On multivariable regression analysis, when controlling for confounding factors, antidepressant use was again associated with a lower likelihood of discharge to home (aOR 0.71, 95\% CI 0.60 to 0.84 , $\mathrm{P}<0.001$ ) (online supplementary table 2 ). Female gender, 
arrival from an acute care facility, diabetes, hypertension, weakness on initial exam, and hospitalisation complicated by pneumonia or urinary tract infection decreased the odds of discharge to home (online supplementary table 2).

\section{DISCUSSION}

In the present study, we explored the impact of antecedent SSRI use on functional status at discharge after AIS. Based on the multiple clinical studies suggesting a benefit for poststroke initiation of an SSRI, ${ }^{810-1223-25}$ our hypothesis was that preSSRI use would portend favourable outcomes. Surprisingly, despite no differences in admission NIHSS score, treatment with intravenous tPA at our facility, endovascular thrombolysis or length of stay, patients taking an SSRI or other antidepressant prior to an AIS were less likely to be sent home or ambulate unassisted on discharge. These findings would suggest that preSSRI/antidepressant use is associated with worse poststroke outcomes, and are in contrast to several studies showing a benefit of poststroke SSRI initiation on motor recovery. ${ }^{812} 24{ }^{26}$ Understanding the mechanism for these observations could prove informative in both advancing our understanding of SSRI-mediated effects on AIS and stroke recovery and targeting rehabilitation efforts in patients with depression.

Our analysis and that of the placebo-controlled study of fluoxetine for motor recovery after AIS (FLAME) have several important differences in terms of study design and patient demographics that merit consideration. ${ }^{8}$ First, in the FLAME trial, subjects were excluded if they had a diagnosis of depression or were taking antidepressant medications in the month prior to their admission. ${ }^{8}$ In contrast, all of our subjects were on SSRIs or other antidepressants prior to their admission and $6.4 \%$ had a diagnosis of depression. Additionally, there was a significant difference in stroke severity between the FLAME trial and our study. In the FLAME trial, the trial design selected for patients with moderate to severe stroke deficits, which resulted in $96 \%-100 \%$ of subjects having a baseline modified Rankin Scale score of 4 or $5 .{ }^{8}$ Our study, on the other hand, had much less severe stroke deficits, illustrated by the median NIHSS score of 4-5 among the groups. The differences in study design and patient demographics of our study and the FLAME trial certainly limit comparisons; however, an outstanding question is how preSSRI use modulates the decreased odds of discharge to home or ambulatory status at discharge.

There are several plausible explanations for our observed results. In our study, patients in the preSSRI or preAD group were more likely to have experienced a prior stroke or TIA and, despite no difference in admission NIHSS score, present with weakness as the initial exam finding. A parsimonious explanation for our findings would be that the preSSRI/preAD groups are biased towards worse motor outcomes given their pre-existing infarct burden and initial presentation with weakness.
In addition, the increased rates of pneumonia in the preSSRI/preAD groups could impact functional status at discharge given the multifactorial inputs that influence ambulatory status. Arguing against these hypotheses, however, there was no difference in the length of stay or admission NIHSS scores between groups.

It is likely that the apparent role of preSSRI or antidepressant use on poststroke recovery is influenced by factors extending from the molecular to psychosocial levels. In animal studies of ischaemic stroke, SSRIs have been demonstrated to profoundly improve neurobehavioural outcomes. ${ }^{13} 14$ The beneficial effects of SSRIs in animals include reduced infarct volume, ${ }^{16}$ upregulated hippocampal neurogenesis ${ }^{15}$ and improved cerebral blood flow autoregulation. ${ }^{17}$ These possible mechanisms of action, at least in part, seem to be mediated by SSRI-induced upregulation of BDNF. ${ }^{18} 1927$ At the neurobehavioural level, poststroke depression is exceedingly common with an estimated prevalence of $9 \%-34 \%^{2829}$ and certainly has the potential to impact participation in acute, intensive rehabilitation efforts. Even prior to AIS, a large systematic meta-analysis showed an association between depression and increased risk of stroke. ${ }^{30}$ Whether preSSRI/antidepressant use is a marker of less favourable outcomes or worse motor deficits warrants further studies.

To better understand how preSSRI use could influence poststroke functional status, future studies should assess the influence on infarct volume and location, perfusion/ diffusion mismatch and changes in BDNF levels in AIS. It has already been reported that in the acute poststroke period, there is a correlation between BDNF levels and functional status at 90 days. ${ }^{31}$ For this reason, it is conceivable that chronic BDNF upregulation from SSRIs could impact the acute/compensatory response of the normal brain to ischaemia.

There are several limitations to this study. First, the assessment of functional outcomes as ambulatory status at discharge and disposition, while both informative markers of functional status, shows each to be influenced by multiple factors that complicate speculation on the underlying cause. Future studies focused on more specific metrics, such as stroke location/subtype, infarct volume, changes in depression severity poststroke and more specific assays of motor weakness (eg, NIHSS motor score), would therefore be useful. Second, the preSSRI/ preAD groups had significantly more prior strokes than the control group. Whether this discrepancy is reflective of the change in clinical practice because of FLAME and other studies ${ }^{8326}$ or the incidence of poststroke depression, there is potential for bias. In the acute stroke period, prior compensated infarct burden could predispose to recrudesce and thereby influence functional status at discharge. Despite this observation, there was no significant difference between groups in ambulatory status on admission. Another limitation is the discordance between patients with a diagnosis of depression and preSSRI use in our study. This observation is likely a reflection of patients taking SSRIs for diseases other than depression and/or 
the relatively poor sensitivity of automated approaches for extracting depression diagnoses from the medical record. ${ }^{32}$ An additional consideration is whether the results of our single-centre analysis are generalisable to the broader population. In comparing our patient population with the nationwide GWTG-Stroke registry, there are several notable differences between our preSSRI population and the nationwide database, including age (68 vs 74 years), white race $(87 \%$ vs $75.1 \%$ ) and discharge to rehabilitation facility ( $46.1 \%$ vs $15.7 \%$ ), which represent important considerations. ${ }^{22}$ Lastly, in this study and data registry, we lack information on the dose and duration of SSRI or antidepressant use, both of which would be informative in interpreting our observed results.

The importance of further investigations into this area is highlighted by the mixed results of clinical studies investigating SSRI use in humans with ischaemic or haemorrhagic stroke. An observational study of preSSRI use showed increased stroke severity and 30-day mortality in patients with haemorrhagic stroke but no association in patients with AIS. ${ }^{20} \mathrm{~A}$ retrospective study on preadmission SSRI or serotonin and norepinephrine reuptake inhibitor (SNRI) use showed higher inhospital mortality in patients admitted to the intensive care unit setting. ${ }^{33}$ A small, retrospective comparison of prestroke versus poststroke SSRI treatment suggested pretreatment was associated with favourable functional outcomes and $\Delta$ NIHSS score at discharge, ${ }^{9}$ although this interpretation was limited by sample size and intergroup differences in initial stroke severity and percentage receiving intravenous thrombolysis.

\section{CONCLUSIONS}

In total, these findings highlight the need for additional clinical studies to elucidate the impact of preSSRI use on stroke outcomes and the underlying mechanism. Our findings suggest that preSSRI or antidepressant use imputes at discharge a decreased likelihood of discharge to home or ability to ambulate independently. Understanding how SSRIs/antidepressants modulate the intrinsic response to ischaemic stroke should positively influence patient care in the peristroke period.

Acknowledgements The authors would like to thank the Partners HealthCare RPDR group for facilitating use of their database.

Contributors MRE was responsible for study design, data acquisition and analysis, interpretation of data, drafting and revising the manuscript, final approval of the version to be published, and agreement to be accountable for all aspects of the work. KAS was responsible for data acquisition and analysis, critical revision of the manuscript for intellectual content, final approval of the version to be published, and agreement to be accountable for all aspects of the work. LHS was responsible for study design, interpretation of data, critical revision of the manuscript for intellectual content, final approval of the version to be published and agreement to be accountable for all aspects of the work.

Competing interests LHS: Chair of the AHA GWTG-Stroke Clinical Workgroup (unpaid), and stroke systems consultant to the Massachusetts Department of Public Health and received grant from its Center for Disease Control's Paul Coverdell Acute Stroke Registry. He serves as a member of the independent international steering committee for the DIAS 3 and 4 trials of desmoteplase for AIS (Lundbeck); of the DSMB for the DeVOTE trial, a clinical trial comparing the cardiovascular safety of insulin degludec with that of insulin glargine in subjects with type 2 diabetes at high risk of cardiovascular events (Novo Nordisk); and the Clinical Event Committee and DSMB for the 3D Separator Trial of endovascular reperfusion in AIS (Penumbra). $\mathrm{He}$ is PI of the NINDS-funded MR Witness trial of extended window, MRE-guided alteplase (NCT01282242) for which Genentech provides alteplase free of charge to MGH for distribution, as well as modest per-patient supplemental site payments. Patient consent Detail has been removed from this case description/these case descriptions to ensure anonymity. The editors and reviewers have seen the detailed information available and are satisfied that the information backs up the case the authors are making.

Ethics approval Massachusetts General Institutional Review Board.

Provenance and peer review Not commissioned; externally peer reviewed. Data sharing statement The authors agree to make available the data used in this analysis for the purposes of reproducing the results and with permission by the local institutional review board.

Open Access This is an Open Access article distributed in accordance with the Creative Commons Attribution Non Commercial (CC BY-NC 4.0) license, which permits others to distribute, remix, adapt, build upon this work non-commercially, and license their derivative works on different terms, provided the original work is properly cited and the use is non-commercial. See: http://creativecommons.org/ licenses/by-nc/4.0/

(c) Article author(s) (or their employer(s) unless otherwise stated in the text of the article) 2018. All rights reserved. No commercial use is permitted unless otherwise expressly granted.

\section{REFERENCES}

1. National Institute of Neurological Disorders and Stroke rt-PA Stroke Study Group. Tissue plasminogen activator for acute ischemic stroke. N Engl J Med 1995;333:1581-7.

2. Hacke W, Kaste M, Bluhmki E, et al. Thrombolysis with alteplase 3 to 4.5 hours after acute ischemic stroke. N Engl J Med 2008;359:1317-29.

3. Berkhemer OA, Fransen PS, Beumer D, et al. A randomized trial of intraarterial treatment for acute ischemic stroke. N Engl J Med 2015;372:11-20.

4. Saver JL, Goyal M, Bonafe A, et al. Stent-retriever thrombectomy after intravenous t-PA vs. t-PA alone in stroke. N Engl J Med 2015;372:2285-95

5. Kelly-Hayes M, Beiser A, Kase CS, et al. The influence of gender and age on disability following ischemic stroke: the Framingham study. $J$ Stroke Cerebrovasc Dis 2003;12:119-26.

6. Feigin VL, Forouzanfar MH, Krishnamurthi R, et al. Global and regional burden of stroke during 1990-2010: findings from the Global Burden of Disease Study 2010. Lancet 2014;383:245-55.

7. Go AS, Mozaffarian D, Roger VL, et al. Executive summary: heart disease and stroke statistics--2014 update: a report from the American Heart Association. Circulation 2014;129:399-410.

8. Chollet F, Tardy J, Albucher JF, et al. Fluoxetine for motor recovery after acute ischaemic stroke (FLAME): a randomised placebocontrolled trial. Lancet Neurol 2011;10:123-30.

9. Siepmann T, Kepplinger J, Zerna C, et al. The effects of pretreatment versus de novo treatment with selective serotonin reuptake inhibitors on short-term outcome after acute ischemic stroke. J Stroke Cerebrovasc Dis 2015;24:1886-92.

10. Mead GE, Hsieh CF, Lee R, et al. Selective serotonin reuptake inhibitors (SSRIs) for stroke recovery. Cochrane Database Syst Rev 2012;11:CD009286.

11. Zittel S, Weiller C, Liepert J. Citalopram improves dexterity in chronic stroke patients. Neurorehabil Neural Repair 2008;22:311-4.

12. Acler M, Robol E, Fiaschi A, et al. A double blind placebo RCT to investigate the effects of serotonergic modulation on brain excitability and motor recovery in stroke patients. J Neurol 2009;256:1152-8.

13. Siepmann T, Penzlin Al, Kepplinger J, et al. Selective serotonin reuptake inhibitors to improve outcome in acute ischemic stroke: possible mechanisms and clinical evidence. Brain Behav 2015;5:e00373.

14. McCann SK, Irvine C, Mead GE, et al. Efficacy of antidepressants in animal models of ischemic stroke: a systematic review and metaanalysis. Stroke 2014;45:3055-63.

15. Schmidt HD, Duman RS. The role of neurotrophic factors in adult hippocampal neurogenesis, antidepressant treatments and animal models of depressive-like behavior. Behav Pharmacol 2007;18:391-418. 
16. Lim CM, Kim SW, Park JY, et al. Fluoxetine affords robust neuroprotection in the postischemic brain via its anti-inflammatory effect. J Neurosci Res 2009;87:1037-45.

17. Shin TK, Kang MS, Lee HY, et al. Fluoxetine and sertraline attenuate postischemic brain injury in mice. Korean $\mathrm{J}$ Physiol Pharmacol 2009;13:257-63.

18. Maya Vetencourt JF, Sale A, Viegi A, et al. The antidepressant fluoxetine restores plasticity in the adult visual cortex. Science 2008;320:385-8.

19. Schäbitz WR, Schwab S, Spranger M, et al. Intraventricular brainderived neurotrophic factor reduces infarct size after focal cerebral ischemia in rats. J Cereb Blood Flow Metab 1997;17:500-6.

20. Mortensen JK, Larsson H, Johnsen SP, et al. Impact of prestroke selective serotonin reuptake inhibitor treatment on stroke severity and mortality. Stroke 2014:45:2121-3.

21. Fonarow GC, Reeves MJ, Smith EE, et al. Characteristics, performance measures, and in-hospital outcomes of the first one million stroke and transient ischemic attack admissions in get with the guidelines-stroke. Circ Cardiovasc Qual Outcomes 2010;3:291-302

22. Schwamm LH, Fonarow GC, Reeves MJ, et al. Get With the Guidelines-Stroke is associated with sustained improvement in care for patients hospitalized with acute stroke or transient ischemic attack. Circulation 2009;119:107-15.

23. Mikami K, Jorge RE, Adams HP, et al. Effect of antidepressants on the course of disability following stroke. Am J Geriatr Psychiatry 2011;19:1007-15.

24. Dam M, Tonin P, De Boni A, et al. Effects of fluoxetine and maprotiline on functional recovery in poststroke hemiplegic patients undergoing rehabilitation therapy. Stroke 1996;27:1211-4.
25. Pariente J, Loubinoux I, Carel C, et al. Fluoxetine modulates motor performance and cerebral activation of patients recovering from stroke. Ann Neurol 2001;50:718-29.

26. Mead GE, Hsieh CF, Lee R, et al. Selective serotonin reuptake inhibitors for stroke recovery: a systematic review and meta-analysis. Stroke 2013;44:844-50.

27. Ploughman $\mathrm{M}$, Windle V, MacLellan CL, et al. Brain-derived neurotrophic factor contributes to recovery of skilled reaching after focal ischemia in rats. Stroke 2009;40:1490-5.

28. Whyte EM, Mulsant BH, Rovner BW, et al. Preventing depression after stroke. Int Rev Psychiatry 2006;18:471-81.

29. Whyte EM, Mulsant BH. Post stroke depression: epidemiology, pathophysiology, and biological treatment. Biol Psychiatry 2002;52:253-64.

30. Barlinn K, Kepplinger J, Puetz V, et al. Exploring the risk-factor association between depression and incident stroke: a systematic review and meta-analysis. Neuropsychiatr Dis Treat 2015;11:1-14.

31. Lasek-Bal A, Jędrzejowska-Szypułka H, Różycka J, et al. Low concentration of BDNF in the acute phase of ischemic stroke as a factor in poor prognosis in terms of functional status of patients. Med Sci Monit 2015;21:3900-5.

32. Fiest KM, Jette N, Quan $\mathrm{H}$, et al. Systematic review and assessment of validated case definitions for depression in administrative data. BMC Psychiatry 2014;14:289.

33. Ghassemi M, Marshall J, Singh N, et al. Leveraging a critical care database: selective serotonin reuptake inhibitor use prior to ICU admission is associated with increased hospital mortality. Chest 2014;145:745-52. 